Filigrane

Écoutes psychanalytiques

\title{
Présentation du numéro
}

\section{Sophie Gilbert}

Volume 26, numéro 2, 2017

URI : https://id.erudit.org/iderudit/1055356ar

DOI : https://doi.org/10.7202/1055356ar

Aller au sommaire du numéro

\section{Éditeur(s)}

Revue Santé mentale au Québec

\section{ISSN}

1192-1412 (imprimé)

1911-4656 (numérique)

Découvrir la revue

\section{Citer ce document}

Gilbert, S. (2017). Présentation du numéro. Filigrane, 26(2), 5-6. https://doi.org/10.7202/1055356ar d'utilisation que vous pouvez consulter en ligne.

https://apropos.erudit.org/fr/usagers/politique-dutilisation/ 


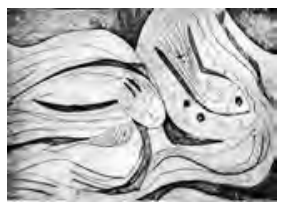

\title{
Présentation du numéro
}

\author{
Sophie Gilbert
}

e numéro fait suite au dossier précédent consacré à «La terreur des l'objet d'un colloque à Montréal à l'automne 2016, se voulait d'abord une allusion au vécu de terreur des enfants victimes de violence ou de négligence. Mais de façon plus subtile, ce thème renvoie à la peur, parfois paralysante ou alors, source d'agirs, issue de la confrontation à la pulsionnalité infantile - en particulier la confrontation de l'adulte, du donneur de soin, et plus largement, de la société. Si le colloque a donné lieu à différentes figurations et élaborations autour de ce thème, il a aussi permis d'en faire ressortir certains éléments fondamentaux. Ce numéro s'amorce donc avec la deuxième et dernière partie de ce dossier, sous l'angle des fondamentaux psychanalytiques suivants: les interdits, le jeu, le traumatisme et la symbolisation.

Dans un premier temps, Maurice Berger nous amène à revisiter la question des interdits. À la suite d'un bref exposé sur les considérations sociétales relatives à ce concept, certains repères sur la fonction et la construction psychique de ceux-ci sont envisagés. Puis, l'auteur aborde, avec moult exemples, la référence aux interdits dans la pratique clinique auprès des enfants et de leurs parents.

Ce dossier s'achève avec une série de deux articles dans lesquels Miguel M. Terradas et ses collaborateurs abordent le travail psychanalytique par le jeu, dans le cas de trauma complexe chez l'enfant. Des réflexions théoriques amènent d'abord le lecteur sur la piste de l'exploration du travail de mentalisation exprimé dans le jeu d'enfant. Puis, l'article suivant est consacré à une riche présentation clinique de cas, illustrative de l'évolution clinique d'un enfant à partir du déploiement de fantasmes et d'angoisses par le jeu.

Par la suite, la rubrique Hétéros accueille un riche article de Nicolas Evzonas sur un thème trop rarement abordé de front en psychanalyse: la jalousie. L'auteur propose un dialogue entre psychanalyse et «clinique artistique» à partir d'œuvres littéraires, théâtrales et cinématographiques, afin de soutenir un vaste portrait de ce concept aux multiples facettes. 
Le dialogue entre psychanalyse et cinématographie est ensuite ouvert différemment, dans notre nouvelle rubrique: "Psychanalyse et cinéma». Dans celle-ci sont présentés des commentaires de films issus de l'activité Ciné-psy organisée régulièrement par l'Association des psychothérapeutes psychanalytiques du Québec (APPQ). Dans ce numéro sont publiés les commentaires de Martin Gauthier et de Louis Pinard, à partir, respectivement, des films «Phoenix» et «Eraserhead».

Ce numéro se termine avec une présentation du livre Lettres du divan, par Louise Grenier, directrice de publication de cet ouvrage collectif.

Sophie Gilbert gilbert.sophie@uqam.ca 